\title{
COVID-19 CARDIAC ARREST DUE TO PRINZMETAL'S ANGINA IN A PREVIOUSLY NORMAL HEART
}

\author{
Melinda Wang ${ }^{1}$, Andrew Talon ${ }^{1}$, and Mehrdad Saririan ${ }^{1}$ \\ ${ }^{1}$ Creighton University School of Medicine Phoenix Regional Campus
}

March 28, 2021

\begin{abstract}
There has been a compelling body of evidence of COVID-19 causing cardiac complications such as myocarditis and increased risk of coronary thrombosis. Acute coronary syndrome due to coronary vasospasm is an unusual occurrence.

\section{Introduction}

Cardiac complications have been found to be prevalent in up to $30 \%$ of severe respiratory syndrome coronavirus 2 (SARS-CoV-2) patients [1]. Although myocarditis has been the presumptive culprit of myocardial injury in the majority of cases, one-third of SARS-CoV-2 patients showing ST-segment elevation on the ECG have been found to have angiographically normal coronary arteries. This event suggests that another mechanism may be due to myocardial injury [2]. We present a case of an acute coronary syndrome due to coronary vasospasm, rather than thrombosis, in a SARS-CoV-2 patient.
\end{abstract}

\section{Case description}

A 62-year-old man diagnosed with COVID-19 pneumonia presented 8 days after his positive COVID test with a chief complaint of worsening dyspnea. He had a prior history of bacterial pneumonia requiring hospitalizations and hypertension. He did not have any cardiac history. On presentation in the ED, the patient was hypoxic with $76 \%$ oxygen saturation on room air and required 15 liters of oxygen/min through a nonrebreather mask to maintain his saturation levels above 90\%. Computed tomography (CT) of the chest was negative for pulmonary embolism. He was treated with dexamethasone $6 \mathrm{mg}$ daily (10-day course), therapeutic dose anticoagulation (enoxaparin $1 \mathrm{mg} / \mathrm{kg}$ ), and oxygen. The patient's daily complaints were dyspnea and cough, and he denied chest pain. On day 3 of his hospitalization, the patient's oxygen saturations decreased and the respiratory therapist was called to his room to increase his oxygen requirement. Before his oxygen could be maximized, he had cardiac arrest with pulseless electrical activity (PEA). Following resuscitation efforts, the patient was intubated and transferred to the intensive care unit. The patient's electrocardiogram was consistent with anterior lead ST-segment elevations (Figure 1). His initial troponin I was $0.012 \mathrm{ng} / \mathrm{ml}$. He was started on aspirin, high-intensity statin, ticagrelor, and low intensity heparin infusion. Urgent coronary angiography revealed multifocal stenosis in the left anterior descending coronary artery (LAD), with myocardial infarction flow 3 (Figure 2). He otherwise had angiographically normal coronary vessels without signs of plaque erosion or rupture. The lesions in the LAD were treated with intracoronary nitroglycerin for suspected coronary spasm, revealing complete resolution of the stenotic segment with normal angiographic appearance of the vessel (Figure 3). Intravascular ultrasound was also performed revealing no significant atherosclerotic disease, remodeling, dissection, or thrombus (Figure 4). The patient's condition did not improve and he was eventually placed on vasopressor support. He soon developed mixed cardiogenic and septic shock with multiorgan system failure. Laboratory examinations revealed D-dimer of $1119 \mathrm{ng} / \mathrm{mL}$. Inflammatory markers including erythrocyte sedimentation rate, ferritin, and C-reactive protein (CRP) were 
not evaluated. Owing to the patient's need for ongoing vasopressor support, he was unable to be started on nitrates or calcium channel blockers.

\section{Discussion}

We diagnosed our patient with coronary vasospasm given the visible resolution of stenosis after intracoronary nitroglycerin. Our workup did not reveal any evidence of coronary artery disease or structural heart disease (Figure 4). A thromboembolic phenomenon would also be unlikely since the patient was receiving therapeutic anticoagulation throughout his hospitalization. Treatment modalities for coronary vasospasm after diagnosis include nitrates or calcium blockers. However, our patient was not a candidate for treatment due to his hemodynamic instability.

Coronary vasospasm is defined as a transient abnormal contraction of the muscle layer of an epicardial coronary artery resulting in myocardial ischemia [1]. Previous cases of ST-segment elevation have shown normal coronary arteries on angiography [2]. In these cases, transient coronary vasospasm should be considered as a cause of the ST-elevation changes seen in COVID-19 patients. It is likely that coronary vasospasm is more prevalent than currently reported in COVID-19. However, most patients do not have the vasospasm captured on cardiac catheterization. Although the pathogenesis is unclear, we postulate that the severe inflammatory response due to COVID-19 may contribute to endothelial dysfunction and provoking a pulseless electrical activity arrest secondary to coronary vasospasm. Our theory is supported by prior studies reporting that the release of inflammatory mediators during hypersensitivity and anaphylactic reactions have been implicated in inducing coronary vasospasm [3]. Hyperventilation is also another cause of coronary vasospasm, and should be considered in COVID-19 patients due to both respiratory distress and anxiety [4].

\section{Conclusion}

COVID-19 causes complications in many body systems and can be a difficult disease to navigate, thus it is important to recognize atypical presentations. Our case highlighted the rare occurrence of coronary vasospasm associated with COVID-19 in an otherwise normal heart that had no evidence of thrombosis or structural disease. It is important to recognize rare causes of cardiac complications that may at first appear to be a ST-elevation MI. In COVID-19 patients with sudden ST elevations, it may be also worthwhile to consider treating prophylactically for coronary vasospasm.

\section{References}

1. Rivero F, Antuña P, Cuesta J, Alfonso F. Severe coronary spasm in a COVID-19 patient [published online ahead of print, 2020 Aug 1].Catheter Cardiovasc Interv. 2020;10.1002/ccd.29056. doi:10.1002/ccd.29056

2. Bangalore S, Sharma A, Slotwiner A, et al. ST-segment elevation in patients with Covid-19 - a case series. N Engl J Med. 2020;382(25):2478-2480

3. Kounis NG: Kounis syndrome (allergic angina and allergic myocardial infarction): a natural paradigm? Int J Cardiol 2006;110:7-14.

4. Freeman LJ, Nixon PGF. Chest pain and the hyperventilation syndrome-some etiologic considerations. Postgrad Med J 1985;61:957-61.

5. Chu G, Zhang G, Zhang Z, Liu S, Wen Q, Sun B: Clinical Outcome of Coronary Stenting in Patients with Variant Angina Refractory to Medical Treatment: A Consecutive Single-Center Analysis. Med Princ Pract 2013;22:583-587. doi: 10.1159/000354290

6. Tavazzi G, Pellegrini C, Maurelli M, et al. Myocardial localization of coronavirus in COVID-19 cardiogenic shock. Eur J Heart Fail. 2020;22(5):911-915

7. Guo T., Fan Y., Chen M. Cardiovascular implications of fatal outcomes of patients with coronavirus disease 2019 (covid-19) JAMA Cardiol. 2020

8. Meune C, Joly LM, Chiche JD, et al. Diagnosis and management of out-of-hospital cardiac arrest secondary to coronary artery spasm. Resuscitation. 2003;58(2):145-152. doi:10.1016/s0300-9572(03)001497 
Figure 1. Electrocardiogram (ECG) showing ST elevation $>0.1 \mathrm{mV}$ in leads V2 through V6, suggesting ST-segment elevation myocardial infarction.

Figure 2. Left heart catheterization pre intracoronary nitroglycerin injection illustrating multiple stenotic lesions in the left anterior descending artery suspected as coronary vasospasms.

LAD - Left anterior descending artery

Figure 3. Left heart catheterization post intracoronary nitroglycerin injection and resolution of the stenotic lesions in the left anterior descending artery.

LAD - Left anterior descending artery

Figure 4. Intravascular ultrasound of the left anterior descending coronary artery without signs of atherosclerotic significant disease or lesions.

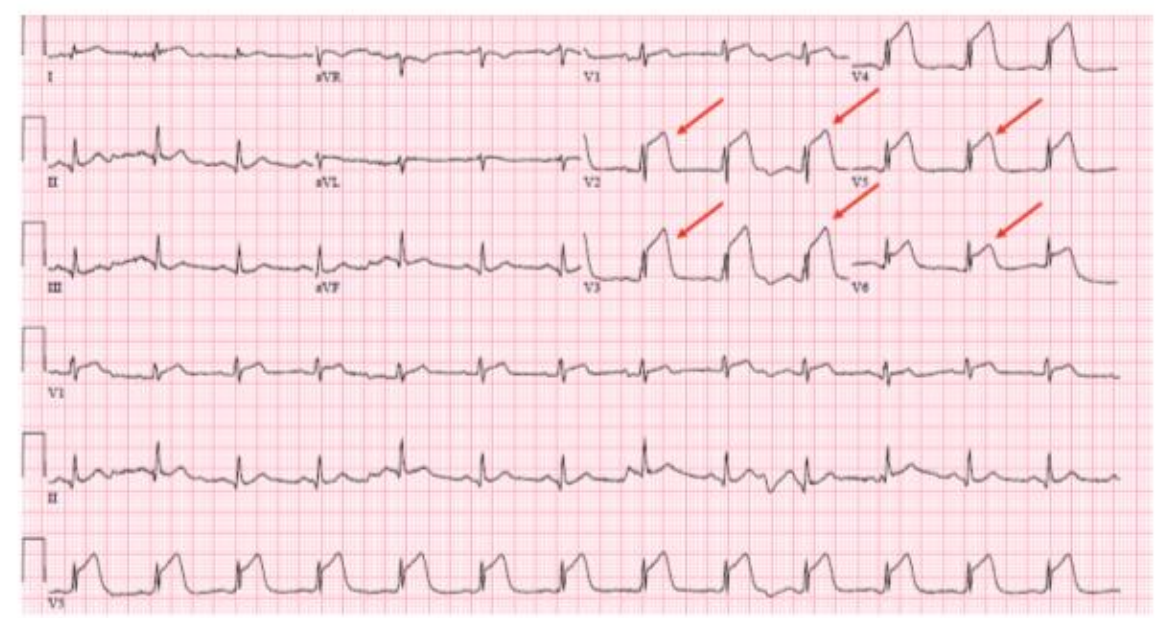




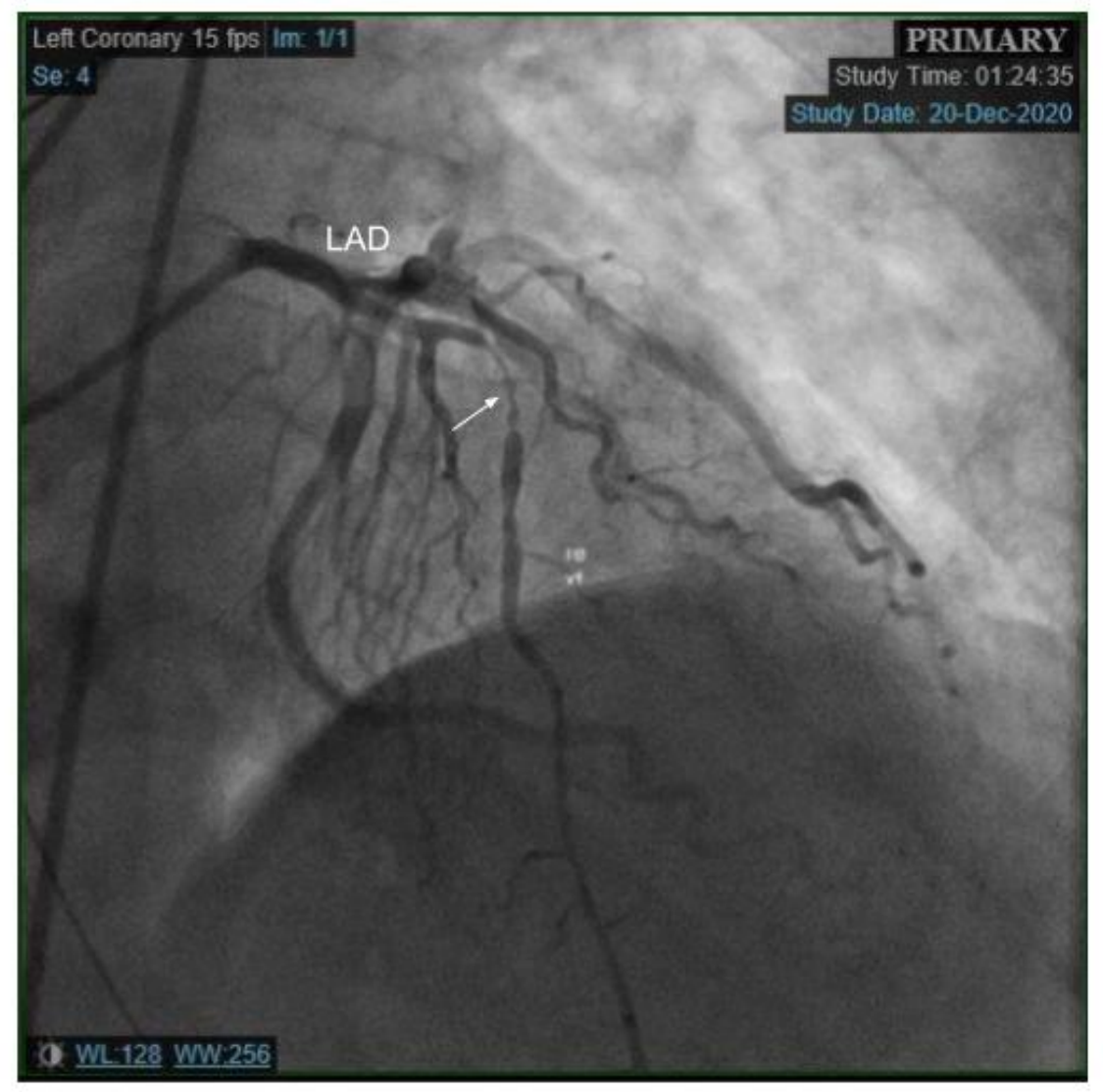




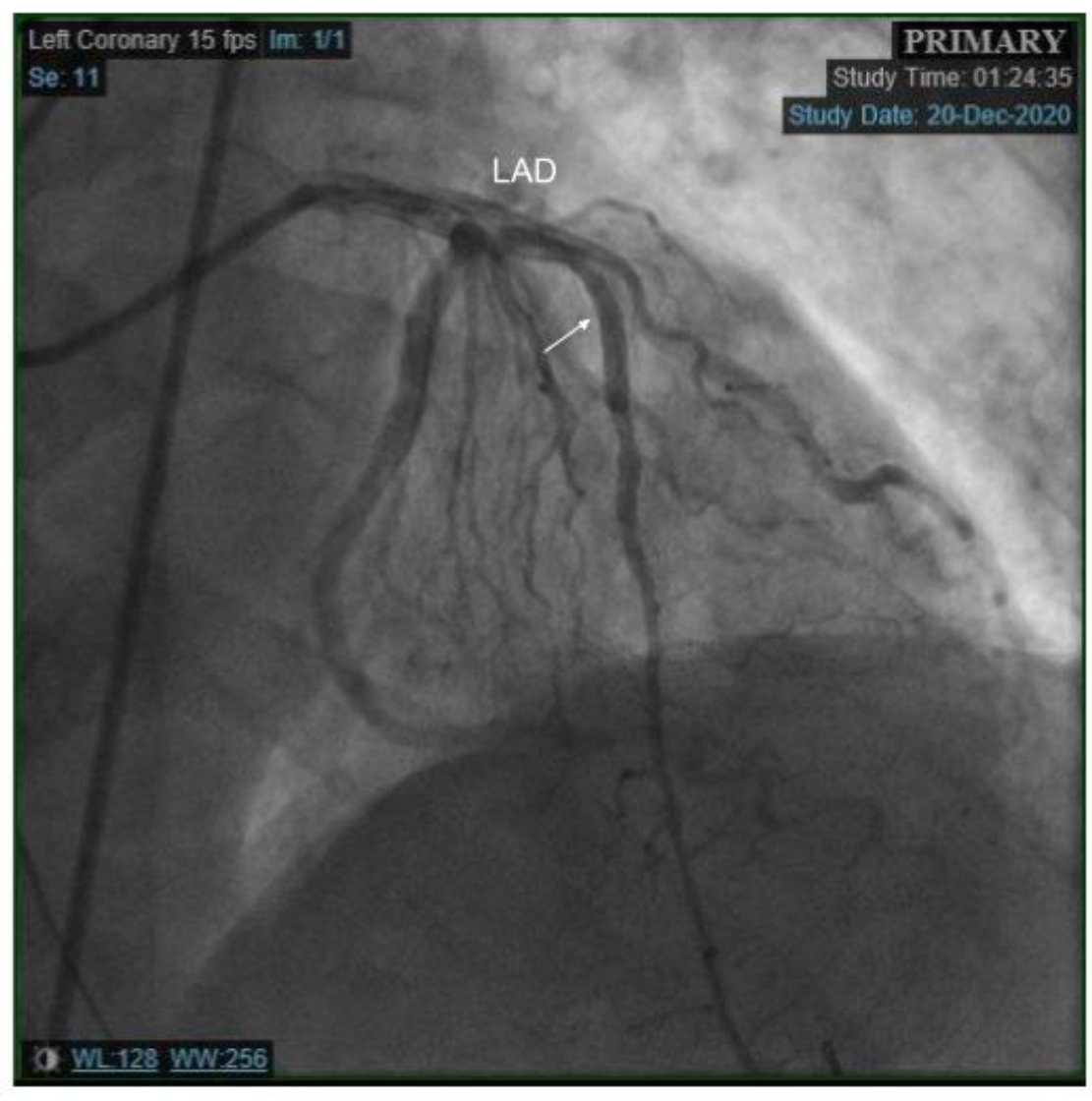




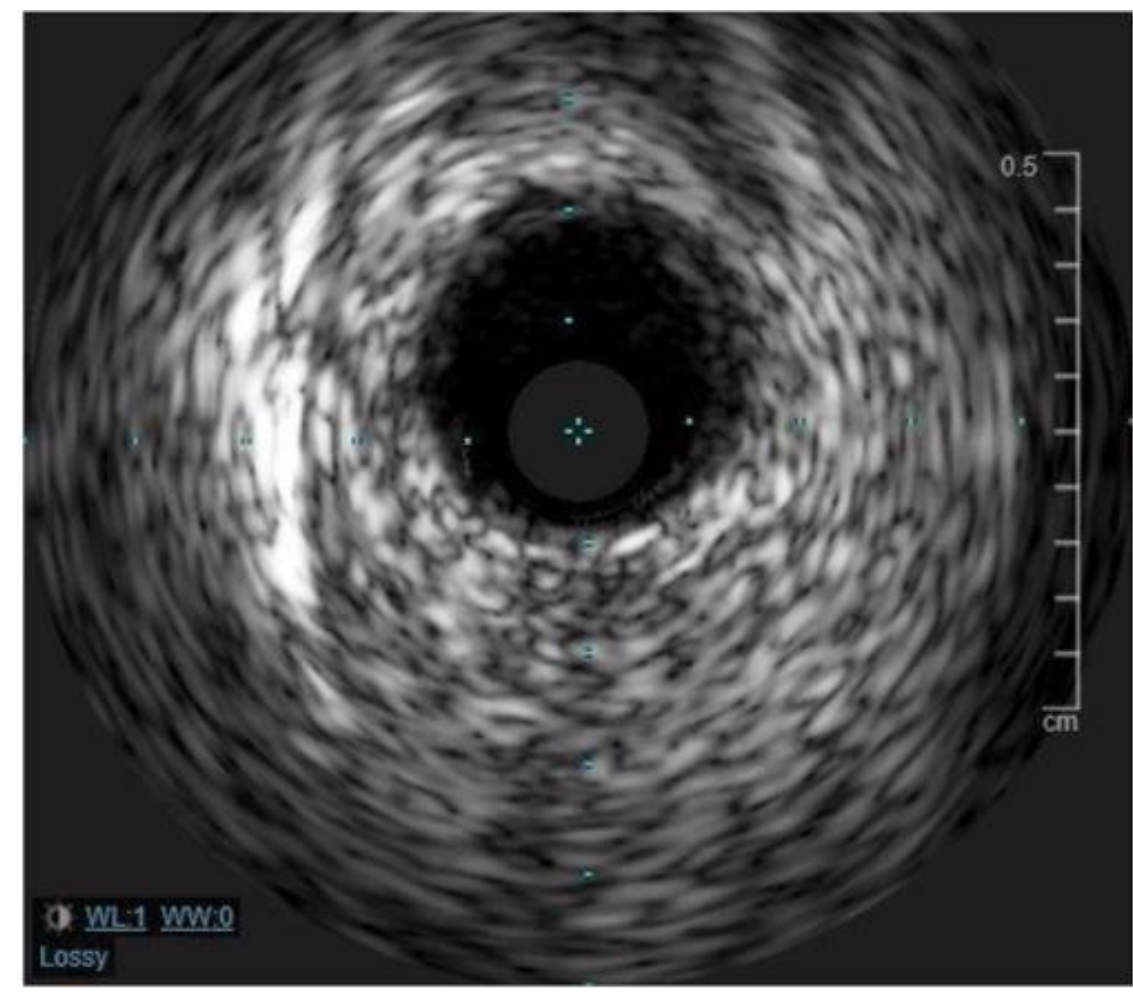

